\title{
Dwell time estimation models for bus rapid transit stations
}

\author{
Fazhi LI*, Zhengyu DUAN, Dongyuan YANG \\ Key Laboratory of Road and Traffic Engineering of the Ministry of Education, School of Transportation Engineering, Tongji Uni- \\ versity, Shanghai 201804, China
}

\begin{abstract}
Bus rapid transit (BRT) systems have been shown to have many advantages including affordability, high capacity vehicles, and reliable service. Due to these attractive advantages, many cities throughout the world are in the process of planning the construction of BRT systems. To improve the performance of BRT systems, many researchers study BRT operation and control, which include the study of dwell times at bus/BRT stations. To ensure the effectiveness of real-time control which aims to avoid bus/BRT vehicles congestion, accurate dwell time models are needed. We develop our models using data from a BRT vehicle survey conducted in Changzhou, China, where BRT lines are built along passenger corridors, and BRT stations are enclosed like light rails. This means that interactions between passengers traveling on the BRT system are more frequent than those in traditional transit system who use platform stations. We statistically analyze the BRT vehicle survey data, and based on this analysis, we are able to make the following conclusions: ( I ) The delay time per passenger at a BRT station is less than that at a non-BRT station, which implies that BRT stations are efficient in the sense that they are able to move passengers quickly. (II) The dwell time follows a logarithmic normal distribution with a mean of 2.56 and a variance of 0.53 . (III) The greater the number of BRT lines serviced by a station, the longer the dwell time is. (IV) Daily travel demands are highest during the morning peak interval where the dwell time, the number of passengers boarding and alighting and the number of passengers on vehicles reach their maximum values. (V) The dwell time is highly positively correlated with the total number of passengers boarding and alighting. (VI) The delay per passenger is negatively correlated with the total number of passengers boarding and alighting. We propose two dwell time models for the BRT station. The first proposed model is a linear model while the second is nonlinear. We introduce the conflict between passengers boarding and alighting into our models. Finally, by comparing our models with the models of Rajbhandari and Chien et al., and TCQSM (Transit Capacity and Quality of Service Manual), we conclude that the proposed nonlinear model can better predict the dwell time at BRT stations.
\end{abstract}

Key words: dwell time model; conflict factor; bus rapid transit (BRT) station

(C) 2012 JMT. All rights reserved.

\section{Introduction}

$\mathrm{I}$ t has been widely recognized that public transportation can effectively relieve traffic congestion in urban areas. Bus rapid transit (BRT) has attracted increasing attention in recent years from both the general public and respective governments as an attractive alternative to other public transportations. In contrast to the traditional bus systems, BRT uses exclusive bus lanes, which guarantees high speed and reliability. However, many factors impact BRT travel time, two of which are the dwell time at bus stops along the route and intersection delays.

The dwell time is the time that a public transportation

Received Nov. 7, 2011; revision accepted Mar. 26, 2012

*Corresponding author. E-mail: fzli1025@126.com (F.Z. LI)

(C) 2012 JMT. All rights reserved

doi: 10.3969/j.issn.2095-087X.2012.03.007 vehicle spends at a station or stop while passengers board and alight. The dwell time therefore depends on the numbers of passengers boarding and alighting, plus other vehicle and stop characteristics such as platform height, door width, fare collection method, internal layout of vehicles, occupancy of vehicles, etc. Traditionally, the dwell time is simply described as a linear function with respect to the number of passengers boarding and alighting. Although linear models are intuitive and simple, they generally can not reach the precision that realtime control and automatic driving require.

In this paper, we consider the relationship between the dwell time and relevant factors, and develop dwell time models for BRT stations.

\section{Literature review}

As of date, there are two aspects of dwell time that are studied. The first considers dwell time models which specify the relationship between dwell time and relevant 
factors, such as the number of passengers boarding and alighting, degree of crowdedness on vehicles, the number of standees on a station platform, etc. These models are typically used to forecast or estimate travel time, and to predict and update bus route logistics. The second aspect considers how the physical environment of transit facilities affects dwell time, including the height of the boarding floor and platform, the height of vehicle floor, the number of doors and their widths, the fare collection method, etc. This research is used to improve transit infrastructure, such as vehicles' door width and platform height. In this paper, we develop a dwell time model, which places our work in the body of research trying to understand relationships between dwell time and various predictors. As such, we briefly review the literature related to dwell time models.

The first dwell time models were typically linear models, which considered only the number of passengers boarding and alighting [1-4]. Lin and Wilson [5] introduced the number of departing standees at a station in the dwell time model for a light rail system.

Another model was proposed by Guenthner and Sinha in 1983, which was per passenger time delay model written as

$$
\frac{D T}{\text { Total }}=5.0-1.2 \ln (\text { Total })
$$

where $D T$ is the dwell time at bus stops, and Total is the total number of passengers boarding and alighting [6].

A relevant nonlinear model was proposed by Puong in 2000, which modeled the effect of the cubic number of standees per door on vehicles to per boarding passenger [7]. Several other nonlinear models were proposed in other studies [8-12].

One of these proposed nonlinear models can be attributed to Gibson and Fernández [8], who proposed a dwell time model:

$$
\begin{aligned}
& D T=\left(\beta_{0}+\beta_{0}^{\prime} \delta_{1}\right)+ \\
& \max _{j=\text { door }}\left\{\left(\beta_{1}+\beta_{1}^{\prime} \delta_{1}+\beta_{1}^{\prime \prime} \delta_{2}\right) P B_{j}+\left(\beta_{2} e^{-\beta_{2}^{\prime} P A_{j}}+\beta_{2}^{\prime \prime} \delta_{3}\right) P A_{j}\right\},
\end{aligned}
$$

where $P B_{j}$ and $P A_{j}$ are the number of passengers boarding and alighting through door $j$, respectively; $\beta_{k}^{i}$ are parameters such that $\beta_{0}^{i}$ is the dead time, $\beta_{1}^{i}$ are per passenger boarding time and $\beta_{2}^{i}$ are per passenger alinghting time (except for $\beta_{2}^{\prime}$, which is the parameter of the exponential function), and $\delta_{k}$ is a dummy variable where $\delta_{1}=1$ if there is congestion on the platform, $\delta_{2}=1$ when more than four passengers board the bus at a single stop, $\delta_{3}=1$ if the aisle of the bus is full, and otherwise, $\delta_{k}$ is zero.
More recently, Jaiswal et al. [11,13-14] introduced time lost into the dwell time model. The time lost by the bus is a loading area specific parameter and is included to account for the requirement that the passenger walk along a lengthy BRT station platform to reach the bus entry door. In their study, they analyzed the differences between boarding and alighting times at three loading areas at one station. As a result of their study, the following conclusions were made: ( I ) per passenger boarding time was $5.9 \mathrm{~s}$ [14], ( II ) the least time lost resulted from the mid-loading (the second) area, while the greatest time lost resulted from loading at the third area [13], and (III) $85 \%$ of the time lost calculated for each of the three loading areas was 7.2, 4.5, and $8.7 \mathrm{~s}$ [11].

Among all the models discussed, the model parameters of TCQSM [4] and Rajbhandari and Chien et al. [9] are obtained easily and these models consider similar predictor variables, so we will compare our model with these two in Section 7.

New technologies, such as automatic driving, realtime control, self-adaptive control, require that we develop quantitatively accurate dwell time models. Though the proposed linear dwell time models have advantages with respect to data collection and computation, their predictions are not accurate enough to keep up with these advancing technologies. These linear models were used in low population areas, like Europe and America, but they may not be ideal for more populated areas, like Asia. In summary, there is an urgent need for new dwell time models.

\section{Definitions}

The dwell time is the time that a public transportion vehicle spends servicing boarding and alighting passengers at a station or a bus stop. The dwell time begins when the public transportation vehicle arrives and stops at station and ends when the vehicle begins to move away from the station. The dwell time is denoted by $D T$.

The numbers of passengers boarding/alighting per door is the number of passengers getting on/off public transport vehicle through a door, and are denoted by $P B_{i}$ and $P A_{i}$, respectively, where $i$ refers to door $i$.

The total numbers of passengers boarding/alighting is the sum of the number of passengers boarding/alighting through all doors. They are denoted by $P B / P A$, where

$$
P B=\sum_{i} P B_{i}, P A=\sum_{i} P A_{i} .
$$

The total number of passengers boarding and alighting is the sum of the number of passengers boarding and alighting through all doors. It is noted by Total, where 
Table 1 Changzhou BRT line system details

\begin{tabular}{ccccccc}
\hline Line & $\begin{array}{c}\text { Length } \\
(\mathrm{km})\end{array}$ & $\begin{array}{c}\text { Average length } \\
\text { of station }(\mathrm{m})\end{array}$ & $\begin{array}{c}\text { Length of } \\
\text { vehicle }(\mathrm{m})\end{array}$ & Door No. & $\begin{array}{c}\text { Departure interval during } \\
\text { peak hours }(\mathrm{min})\end{array}$ & $\begin{array}{c}\text { Departure interval during } \\
\text { non-peak hours }(\mathrm{min})\end{array}$ \\
\hline B1 & 24.5 & 980 & 18 & 4 & $2-3$ & 4 \\
B11 & 17.5 & 693 & 12 & 2 & $3-4$ & $4-6$ \\
B12 & 13.8 & 552 & 12 & 2 & $3-4$ & $5-6$ \\
B13 & 13.0 & 684 & 12 & 2 & $2-3$ & $4-6$ \\
B2 & 20.3 & 781 & 18 & 4 & $4-5$ & $6-8$ \\
B21 & 9.3 & 715 & 18 & 4 & 6 & $6-8$ \\
B22 & 6.4 & 711 & 12 & 2 & $4-5$ & $5-7$ \\
B23 & 13.9 & 463 & 12 & 2 & $3-4$ & $4-6$ \\
\hline
\end{tabular}

$$
\text { Total }=\sum_{i}\left(P B_{i}+P A_{i}\right)
$$

The number of passengers in a public transportion vehicle is the total number of people in the vehicle when it leaves from the upstream station. This variable is denoted by $P V$.

The conflict factor is a variable which describes the interaction between the passengers on the vehicle with those not on the vehicle. It is denoted by $C F$, where

$$
C F_{i}=P B_{i} \times P A_{i},
$$

The delay time per passenger is the average time that every passenger spends boarding and alighting. It is denoted by $D p$, where $D p=D T /$ Total .

\section{Data collection and data processing}

Data was collected in Changzhou city, which is located in east China, and has a population of 5.77 million people as of 2009. In Changzhou, 18\% commuter trips were completed using public transit. As opposed to the conventional routes, in Changzhou BRT vehicles operate on exclusive bus lanes.

Currently, there are two hybrid BRT channels in Changzhou City. The BRT1 channel is composed of main-line B1 and sub-lines B11, B12 and B13. The BRT2 channel is composed of main-line B2 and sublines B21, B22 and B23. These two channels form crossing passenger corridors, where BRT1 runs from south to north and BRT2 from east to west. Every BRT station has two loading areas. The details of these BRT lines are listed in Table 1.

B1 and B2 are main lines of the BRT network. All stations for these main lines are BRT stations, and each station has two loading areas. The height of platforms is level with the BRT vehicles' floors. Passengers prepay their fare at stations' entrance using coins or intelligent card. Passengers can get on or off the BRT using any door of BRT vehicle. Each station services one line or more lines.

Data was collected on board transit survey that took place on June 10, 2010, where 150 volunteers participated in this survey. The $D T$ at the station and the numbers of $P B$ and $P A$ per door were recorded. This survey took place during the hours of 6:30 AM to 6:00 PM. From this survey, we obtained 5958 records, the details of which are shown in Table 2.

Table 2 Summary of the numbers of collected samples

\begin{tabular}{ccc}
\hline Category & $\begin{array}{c}\text { Number of } \\
\text { records }\end{array}$ & $\begin{array}{c}\text { Percent } \\
(\%)\end{array}$ \\
\hline Total & 5958 & 100 \\
B1 & 792 & 13.3 \\
B11 & 860 & 14.4 \\
B12 & 846 & 14.2 \\
B13 & 665 & 11.2 \\
B21 & 864 & 14.5 \\
B22 & 539 & 9.0 \\
B23 & 384 & 6.4 \\
Noon interval & 1008 & 16.9 \\
Morning peak interval & 1849 & 31.0 \\
Evening peak interval & 2372 & 39.8 \\
BRT station, 4-door vehicle & 2039 & 34.2 \\
Non-BRT station,4-door vehicle & 156 & 2.6 \\
BRT station, 2-door vehicle & 1158 & 19.4 \\
Non-BRT station, 2-door vehicle & 2601 & 43.7 \\
A BRT station sharing 1 line & 816 & 13.7 \\
A BRT station sharing 2 lines & 899 & 15.1 \\
BRT station sharing 3 lines & 984 & 16.5 \\
\hline BRT station sharing 4 and 5 lines & 498 & 8.4 \\
\hline
\end{tabular}


Each vehicle on the B1, B2 and B21 lines has four doors while all other vehicles servicing other lines have two doors. One person was assigned to record the number of $P B, P A$ and $D T$ for a single door. So for each BRT station there are four or two $D T$ records. To analyze data and develop our dwell time model, we need to choose one dwell time record per stop. To process the data, we followed two steps.

Step 1: Filtering data. Dwell time data whose value was larger than $180 \mathrm{~s}$ or smaller than $3 \mathrm{~s}$ were deleted. These unusually small or large measurements are likely the result of subjective judgment error and recording error.

Step 2: Determining the dwell time per stop. After step 1, there still exists more than one dwell time record for each stop, so we need to determine which record is to use as our dwell time. The record whose value is clos- est to the mean of all records is chosen as the final dwell time. If there is more than one final dwell time record, we keep the largest as the final dwell time.

\section{Data analysis}

\subsection{Statistical analysis of the BRT system}

\subsubsection{Dwell time difference between BRT stations and non-BRT stations}

Some sub-line stations are not exclusive BRT stations and service both the BRT bus as well as the traditional bus. According to our data analysis (Table 3), the mean dwell time at BRT stations was $16.8 \mathrm{~s}$, which is $4.14 \mathrm{~s}$ longer than that of non-BRT stations, i.e., $33 \%$ of the

Table 3 Statistics of dwell time attributes for BRT and non-BRT stations

\begin{tabular}{|c|c|c|c|c|c|c|}
\hline & \multirow{2}{*}{ Category } & \multirow{2}{*}{ Mean } & \multicolumn{2}{|c|}{$95 \%$ confidence interval for mean } & \multirow{2}{*}{$5 \%$ trimmed mean } & \multirow{2}{*}{$\begin{array}{l}\text { Std. } \\
\text { Dev. }\end{array}$} \\
\hline & & & Lower bound & Upper bound & & \\
\hline \multirow{2}{*}{$D T(\mathrm{~s})$} & BRT station & 16.80 & 16.46 & 17.14 & 15.64 & 9.781 \\
\hline & Non-BRT station & 12.66 & 12.36 & 12.96 & 11.79 & 7.949 \\
\hline \multirow{2}{*}{$\begin{array}{c}D p \\
(\mathrm{~s} / \mathrm{pax})\end{array}$} & BRT station & 2.56 & 2.45 & 2.66 & 2.10 & 2.975 \\
\hline & Non-BRT station & 3.28 & 3.17 & 3.39 & 2.93 & 2.782 \\
\hline \multirow{2}{*}{$\begin{array}{l}\text { Total } \\
(\operatorname{pax})\end{array}$} & BRT station & 11.52 & 11.18 & 11.86 & 10.61 & 9.692 \\
\hline & Non-BRT station & 6.04 & 5.77 & 6.30 & 5.14 & 7.075 \\
\hline \multirow{2}{*}{$\begin{array}{c}P V \\
(\operatorname{pax})\end{array}$} & BRT station & 41.03 & 40.15 & 41.92 & 39.45 & 25.550 \\
\hline & Non-BRT station & 27.61 & 26.87 & 28.35 & 26.07 & 19.877 \\
\hline
\end{tabular}

mean dwell time of non-BRT stations. The average delay time per passenger on a BRT station was $2.56 \mathrm{~s}$, which is $0.72 \mathrm{~s}$ less than that at a non-BRT station, i.e., $22 \%$ of that at a non-BRT station. The average total number of boarding and alighting passengers at BRT stations is 1.9 times that at non-BRT stations. The average number of passengers in a public transportation vehicle at BRT stations is 1.5 times that of non-BRT stations.

By the above comparison, we conclude that the BRT station is more high-efficient than a non-BRT station. BRT stations can reduce trip time and improve the level of services available on that transit system. The BRT dwelling time problem has some new characteristics, such as per passenger delay time, total number of boarding and alighting passengers and load on vehicles, and therefore it is necessary to study the relationship between dwell time and station properties.

\subsubsection{Distribution of dwell time}

The results from statistical data analysis are shown in
Table 4 and Fig. 1. The mean dwell time of BRT in Changzhou was $14.78 \mathrm{~s}$. The dwell time measurements for both the entire data set including all lines and for each BRT line consistently follow the logarithmic normal distribution and the distribution parameters $m u$ and sigma of the data set including all lines were 2.56 and 0.53 .

\subsection{Statistic analysis of BRT station data}

\subsubsection{Time interval analysis}

To understand how dwell time changes throughout different time intervals, we divided the data into three time intervals: the morning peak interval (6:30-9:00), the noon interval (9:00-16:00), and the evening peak interval (16:00-18:00). The results of the statistical analysis of the divided data are displayed in Table 5 and Fig. 2. The maximum mean dwell time occurred in the morning peak interval was $19.03 \mathrm{~s}$. The minimum mean dwell time occurred in the noon interval was $15.61 \mathrm{~s}$. In the evening peak interval, the mean of dwell time was $16.59 \mathrm{~s}$. 


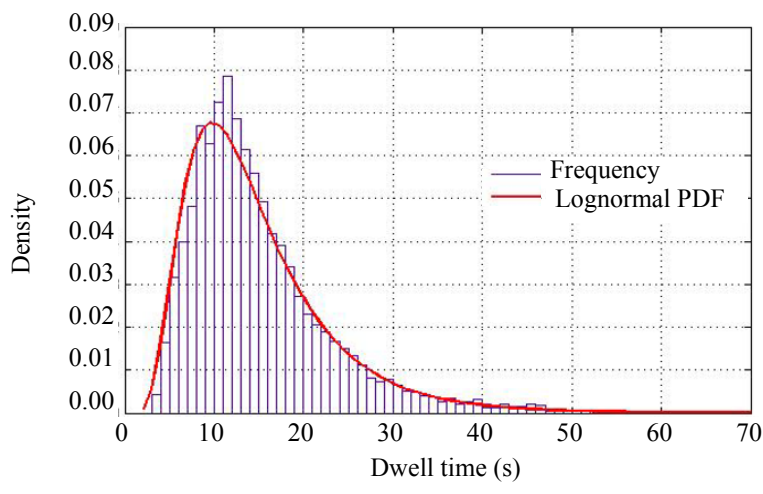

(a) Probability density distribution

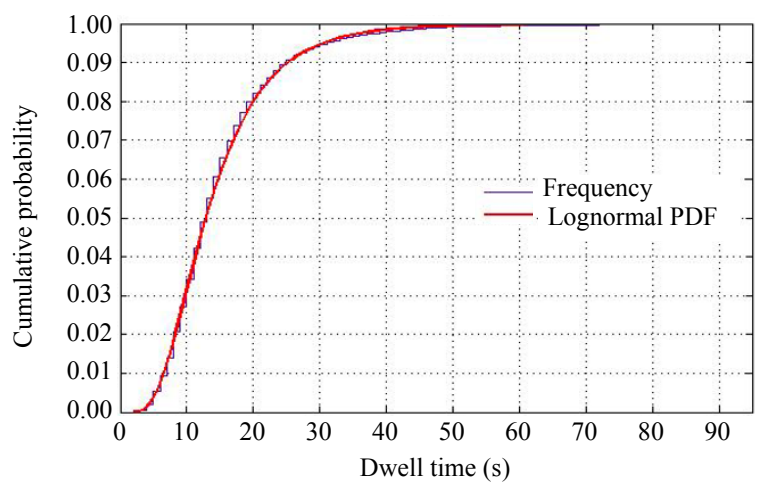

(b) Cumulative probability distribution

Fig. 1 Distributions of dwell time

Table 4 The dwell time distribution parameters for all BRT lines

\begin{tabular}{|c|c|c|c|c|c|c|c|c|c|c|}
\hline \multicolumn{2}{|c|}{ Parameter } & All & B1 & B2 & B11 & B12 & B13 & B21 & B22 & B23 \\
\hline \multicolumn{2}{|c|}{ Mean (s) } & 14.78 & 16.62 & 15.57 & 15.36 & 13.79 & 11.91 & 16.05 & 15.37 & 13.72 \\
\hline \multicolumn{2}{|c|}{ Variance $\left(\mathrm{s}^{2}\right)$} & 69.08 & 63.53 & 40.87 & 75.11 & 90.01 & 54.22 & 45.50 & 50.41 & 65.60 \\
\hline \multirow{2}{*}{$\begin{array}{l}\text { Lognormal } \\
\text { distribution }\end{array}$} & $m u$ & 2.556 & 2.707 & 2.667 & 2.594 & 2.430 & 2.316 & 2.694 & 2.636 & 2.469 \\
\hline & sigma & 0.524 & 0.455 & 0.395 & 0.526 & 0.623 & 0.569 & 0.403 & 0.440 & 0.547 \\
\hline \multirow{2}{*}{ Std. Err. } & $m u$ & 0.007 & 0.016 & 0.013 & 0.018 & 0.021 & 0.022 & 0.022 & 0.022 & 0.017 \\
\hline & sigma & 0.005 & 0.011 & 0.010 & 0.013 & 0.015 & 0.016 & 0.016 & 0.016 & 0.012 \\
\hline
\end{tabular}

Table 5 Statistical analysis according to time interval

\begin{tabular}{|c|c|c|c|c|c|}
\hline Attributes & Time & Mean & Std. Dev. & Skewness & Kurtosis \\
\hline \multirow{3}{*}{$D T(\mathrm{~s})$} & $6: 30-9: 00$ & 19.03 & 10.93 & 2.587 & 9.995 \\
\hline & 9:00-16:00 & 15.61 & 7.89 & 2.999 & 15.942 \\
\hline & $16: 00-18: 00$ & 16.59 & 9.58 & 3.339 & 17.502 \\
\hline \multirow{3}{*}{ Total (pax) } & $6: 30-9: 00$ & 14.34 & 10.87 & 1.198 & 1.282 \\
\hline & $9: 00-16: 00$ & 9.81 & 7.84 & 1.578 & 3.273 \\
\hline & $16: 00-18: 00$ & 12.20 & 9.71 & 1.515 & 2.932 \\
\hline \multirow{3}{*}{$P B($ pax $)$} & $6: 30-9: 00$ & 7.37 & 7.55 & 1.678 & 3.487 \\
\hline & $9: 00-16: 00$ & 5.02 & 5.22 & 1.577 & 2.967 \\
\hline & $16: 00-18: 00$ & 5.77 & 6.86 & 2.100 & 5.886 \\
\hline \multirow{3}{*}{$P A($ pax $)$} & $6: 30-9: 00$ & 6.96 & 7.85 & 1.936 & 5.031 \\
\hline & $9: 00-16: 00$ & 4.79 & 5.26 & 2.330 & 9.740 \\
\hline & $16: 00-18: 00$ & 6.43 & 6.33 & 1.507 & 2.675 \\
\hline \multirow{3}{*}{$D p(\mathrm{~s} / \mathrm{pax})$} & $6: 30-9: 00$ & 2.21 & 2.57 & 6.347 & 72.287 \\
\hline & 9:00-16:00 & 2.85 & 3.09 & 3.301 & 14.271 \\
\hline & 16:00-18:00 & 2.48 & 3.03 & 4.216 & 25.235 \\
\hline \multirow{3}{*}{$P V($ pax $)$} & $6: 30-9: 00$ & 51.67 & 28.74 & 0.699 & 0.464 \\
\hline & 9:00-16:00 & 32.55 & 18.51 & 0.571 & -0.259 \\
\hline & $16: 00-18: 00$ & 43.71 & 25.38 & 0.791 & 0.644 \\
\hline
\end{tabular}




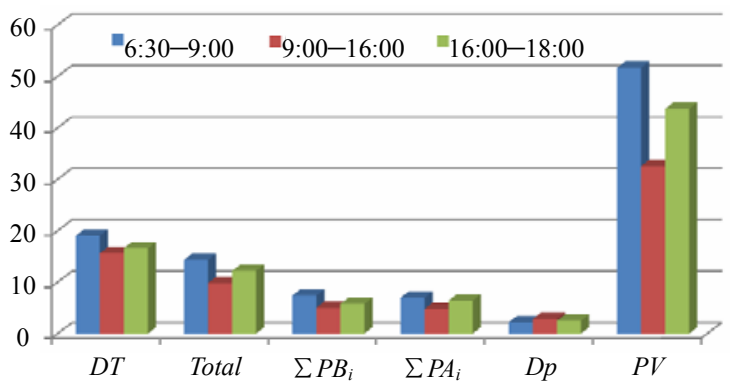

Fig. 2 Statistical analysis according to time interval

The maximum average number of passengers on the BRT vehicle occurred during 6:30-9:00 was 52 persons. The mean number of passengers was smallest during the noon interval and was 33 persons. During 16:00-18:00 interval, the mean number of passengers was 44 persons. In addition, the maximum average number of total on and off passengers was 14 persons during the morning peak.

During the morning peak interval, $D T$, Total, $P B, P A$ and $P V$, are higher than during the other intervals. This means that the transit demand during the morning peak is higher than that of the other intervals, which is in line with our understandings of the BRT. We found that $D T$ was positively correlated with Total, $P B, P A$ and $P V$. Also, when the number of passengers increased, we found that $D p$ decreased. Therefore $D p$ is negatively correlated with Total, $P B, P A$ and $P V$.

\subsubsection{Vehicle type analysis}

There are two types of vehicles servicing the Changzhou BRT system: a 2-door vehicle and a 4-door vehicle. Cross analysis on vehicle types and time intervals was performed for each of DT, Total, $P B, P A, D p$, and $P V$ and the results are shown in Figs. 3-5.

During the morning peak interval, the DT of the 4door vehicles was $17.75 \mathrm{~s}$, and that of 2-door vehicle was $21.18 \mathrm{~s}$. This suggests that 4 -door vehicles are more efficient than 2-door vehicles. The 4-door vehicles service 14.56 passengers on average at each stop, while the 2-door vehicles service 13.96 passengers on average at each stop. Also, the 4-door vehicles are able to carry

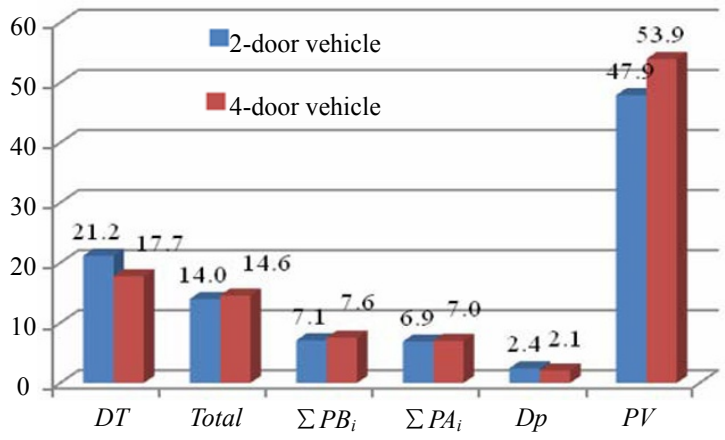

Fig. 3 Analysis of vehicle type during 6:30-9:00

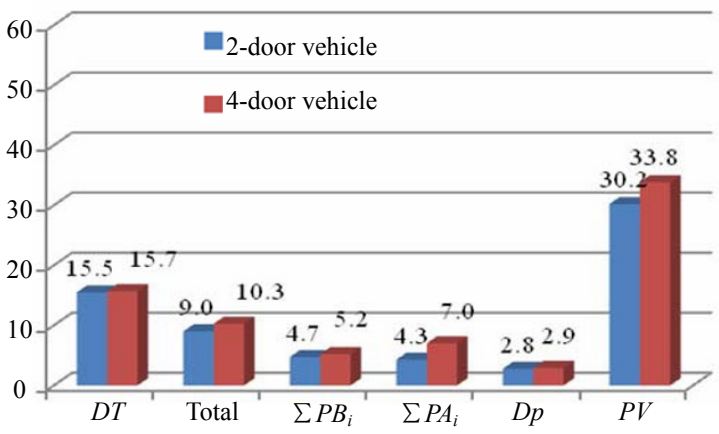

Fig 4 Analysis of vehicle type during 9:00-16:00

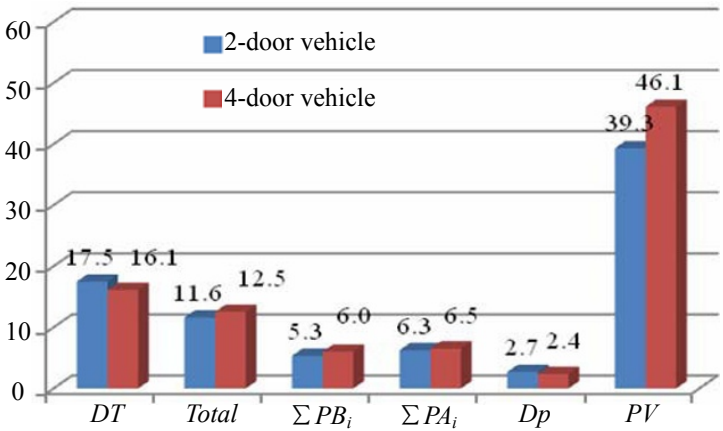

Fig 5 Analysis of vehicle type during 16:00-18:00

more passengers than the 2-door vehicles. Lastly, we note that the $D p$ of the 4-door vehicles was $2.06 \mathrm{~s}$ and that of the 2-door vehicles was $2.45 \mathrm{~s}$, demonstrating that 4-door vehicles are more efficient than 2-door vehicles.

During the noon interval, the $D T$ and $D p$ of the 2door vehicles were smaller than those of the 4-door vehicles. By contrast, it is more efficient for large capacity vehicles to service areas with high-travel demand and for common vehicles to run on low-travel demand transit lines. BRT lines are always built along public transportation corridor. Generally, BRT systems need to carry many passengers, so the 4-door high-capacity vehicle is usually the preferred choice of vehicle.

\subsection{Statistical analysis of the BRT main-lines}

The number of lines that one station services will influence the dwell time and the associated dwell time factors. The number of lines serviced per station ranges from 1 to 5. First we analyze the data from the entire day, and the results are shown in Table 6 . The results demonstrate that the more lines a BRT station services, the longer the $D T$.

In order to further understand the relationships between $D T$ and the factors of influence, we conducted cross analysis with respect to the number of lines that a station services and time interval. The results for the morning peak interval are shown in Fig. 6, those from the noon interval in Fig. 7, and those from the evening 
peak interval in Fig. 8. A comparison of the $D T$ according to the number of the lines that a station services for the three intervals is shown in Fig. 9.

The areas in which a station services many bus lines are generally passenger corridors and central districts of cities, where the demand for public transportation is high. The greater the number of lines that shares a station, the greater the number of passengers per vehicle. The figures show that the DT of BRT stations increases with the number of the BRT lines. Additionally, we also found positive correlations between $D T$ and $P B, P A$ and $P V$.

In Fig. 9, we can see that the dwell time during the evening peak is similar to that during the noon interval. For both of these intervals, the dwell time increases with the number of lines until this number reaches 5 .

Table 6 Statistics by the number of station servicing BRT lines

\begin{tabular}{cccccc}
\hline Attributes & 1 & 2 & 3 & 4 & 5 \\
\hline$D T(\mathrm{~s})$ & 15.11 & 16.33 & 16.54 & 20.27 & 19.69 \\
Total (pax) & 10.54 & 12.62 & 10.64 & 21.46 & 15.30 \\
$P B($ pax $)$ & 5.37 & 6.42 & 5.41 & 9.99 & 7.73 \\
$P A($ pax $)$ & 5.17 & 6.20 & 5.23 & 11.48 & 7.57 \\
$D p(\mathrm{~s} / \mathrm{pax})$ & 2.99 & 2.24 & 2.57 & 1.26 & 1.79 \\
$P V(\operatorname{pax})$ & 37.09 & 43.84 & 49.93 & 46.45 & 52.56 \\
\hline
\end{tabular}

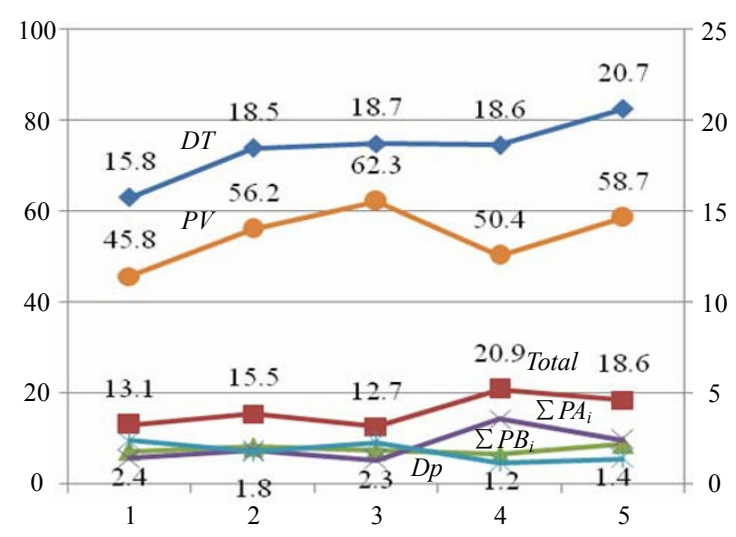

Fig. 6 Morning peak interval (6:30-9:00)

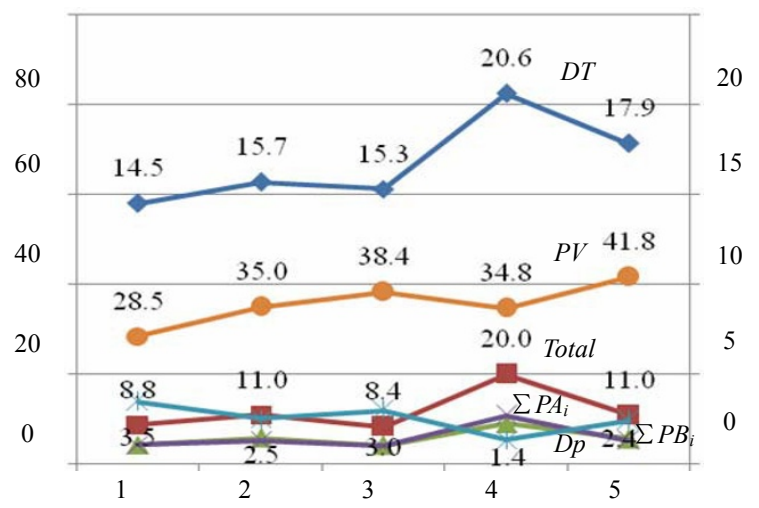

Fig. 7 Noon interval (9:00-16:00)

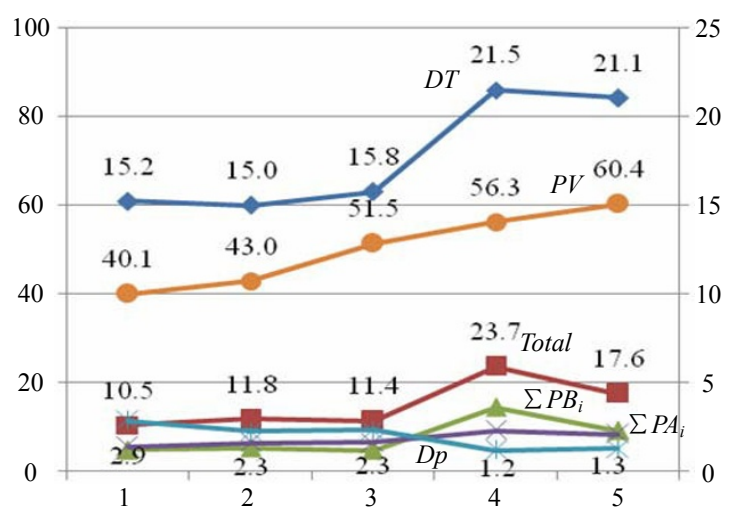

Fig. 8 Evening peak interval (16:00-18:00)

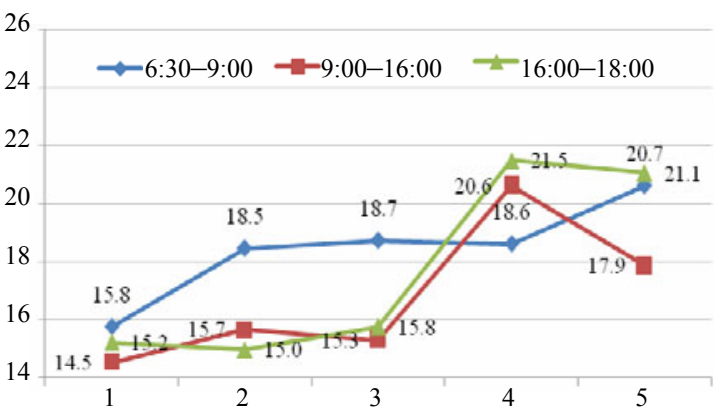

Fig. $9 D T$ comparison of three intervals

Six conclusions can be made as a result of our data analysis: ( I ) The $D p$ at a BRT station is less than that at a non-BRT station, which implies that BRT stations are efficient in the sense that they are able to move passengers quickly. (II) The DT follows a logarithmic normal distribution with a mean of 2.56 and a variance of 0.53 . (III) The greater the BRT lines serviced by a station, the longer the $D T$. (IV) Daily travel demands are highest during the morning peak interval where $D T, P B, P A$ and $P V$ reach their maximum values. (V) The $D T$ is highly positively correlated with $P B, P A$ and $P V$. (VI) The $D p$ is negatively correlated with the total number of passengers boarding and alighting.

\section{Dwell time model}

Traditionally dwell time refers to the time that it takes passengers to board and alight plus the dead time which results from the opening and closing of vehicle doors. Early dwell time models primarily considered the linear relationship between the dwell time and the number of passengers boarding and alighting. Later studies introduced new factors into their dwell time models, such as the number of standing commuters on both vehicles and platforms.

There is no doubt that the components of dwell time are directly linked to the time of passengers boarding 
and alighting plus the dead time. Some scholars have considered dead time to be constant, which is equal to the minimum dwell time. The per passenger delay is associated with many factors, such as vehicle crowdedness, conflict between boarding passengers and alighting passengers, fare payment methods, door width, etc.

The BRT main line stations are level with loading areas, and passengers prepay their fare at the station entrances. In the following models, we consider the time intervals, boarding passengers, alighting passengers, load on vehicles, and conflict factor.

We hypothesize that the load of vehicle can affect per passenger delay and it is a piecewise function with respect to per passenger delay. When it exceeds a threshold, load of vehicle will influence the dwell time, or it will not influence the dwell time.

The correlations between $D T$ and $P B, P A, P V$ and $C F$ are shown in Table 7. The table shows that the most significant correlation is that between $D T$ and $C F$, and therefore it is reasonable to introduce $C F$ into our dwell time models. The correlation coefficient between $D T$ and all other variables is greater than 0.33 , which means that $D T$ may interact with these other variables. Based on these observations, our first model is written as

$$
\begin{aligned}
& D T=t_{0}+t_{b} P B+t_{a} P A+\delta_{1} t_{v}\left(P V-N_{\text {seat }}\right)+ \\
& t_{c} C F+\varepsilon_{1},
\end{aligned}
$$

where $t_{0}, t_{b}, t_{a}, t_{v}$ and $t_{c}$ are parameters; $N_{\text {seat }}$ is the number of seats in the BRT vehicle; $\delta_{1}$ is a dummy viable, equal to 1 for $P V$ above a critical value, otherwise equal to 0 ; $\varepsilon_{1}$ is the model error.

Table 7 Correlations between variables

\begin{tabular}{cccccc}
\hline & $D T$ & $P V$ & $P B$ & $P A$ & $C F$ \\
\hline$D T$ & 1.000 & 0.402 & 0.407 & 0.334 & 0.460 \\
$P V$ & 0.402 & 1.000 & 0.468 & 0.253 & 0.451 \\
$P B$ & 0.407 & 0.468 & 1.000 & -0.01 & 0.555 \\
$P A$ & 0.334 & 0.253 & -0.01 & 1.000 & 0.560 \\
$C F$ & 0.460 & 0.451 & 0.555 & 0.560 & 1.000 \\
\hline
\end{tabular}

Many factors such as $C F$ and $P V$ directly impact the time of per passenger boarding and/or alighting, and therefore indirectly affect dwell time [8]. Also, dwell time is related to the squares of both $P B$ and $P A$ [10]. Combining these results with the model structure of TCQSM [4], model 2 is written as

$$
\begin{gathered}
D T=t_{0}+\max _{i=\text { door }}\left\{\left[t_{b 1}+\delta_{1} t_{b 2}\left(P V-N_{\text {seat }}\right)+t_{b 3} e^{-P B_{i}}+t_{b 4} C F_{i}\right]\left(P B_{i}+t_{b 5} P B_{i}^{2}\right)+\right. \\
\left.\left[t_{a 1}+\delta_{1} t_{a 2}\left(P V-N_{\text {seat }}\right)+t_{a 3} e^{-P A_{i}}+t_{a 4} C F_{i}\right]\left(P A_{i}+t_{a 5} P A_{i}^{2}\right)\right\}+\varepsilon_{2},
\end{gathered}
$$

where $t_{i j}, i \in\{b, a\}$ and $j \in\{1,2,3,4,5\}$, are parameters; are parameters; and $\varepsilon_{2}$ is model error.

\section{Comparison with other models}

To compare and validate the proposed models, referred to as model 1 and model 2, we compared two published dwell time models with our models. One of the models that we compare our models with is referred to as model 3, and was proposed by Rajbhandari and his partners [9]. Model 3 is given by

$$
D T=t_{0}+b \text { Total }+c S T \text { Tolal, }
$$

where $S$ is standees on vehicles.

The second model we compare our results with is referred to as model 4, which was published by TCQSM [4]. Model 4 is given by

$$
D T=t_{0}+t_{b} P B+t_{a} P A .
$$

The data from the morning peak interval (6:30-9:00) for both B1 and B2 was used to fit models 1-4. As in the previous BRT main line analysis, we consider the four cases where a BRT station services one, two, three and four BRT lines. We consider the vehicle to be crowded if there are at least 9 passengers standing in the vehicle [9].
According to the specified capacity of 45 passengers per vehicle, we define as the vehicle to be crowd when the $P V$ exceeds 55 passengers. This means that a vehicle is crowded when there are no less than 10 standing passengers. Then the value of $\delta_{1}$ is

$$
\delta_{1}=\left\{\begin{array}{l}
1, \quad P V \geq 55, \\
0, \quad P V<55 .
\end{array}\right.
$$

For the case when $P V \geq 55, R^{2}$ values are shown in Table 8, and fitness parameters are shown in Tables 10-13.

Table $8 R^{2}$ values for each of four models $(P V \geq 55)$

\begin{tabular}{cccccc}
\hline Models & 1 & 2 & 3 & 4 & All data \\
\hline 1 & 0.485 & 0.344 & 0.428 & 0.444 & 0.421 \\
2 & 0.681 & 0.377 & 0.499 & 0.831 & 0.470 \\
3 & 0.568 & 0.327 & 0.450 & 0.427 & 0.472 \\
4 & 0.356 & 0.247 & 0.232 & 0.252 & 0.294 \\
\hline
\end{tabular}

The ordering of the $R^{2}$ values for the four models for all data is model $3>$ model $2>$ model $1>$ model $4 . R^{2}$ value of model 2 is 0.470 which is very close to the model $3 R^{2}$ value of 0.472 . When we group the data according to the number of lines serviced by a station, the $R^{2}$ values are highest when using model 2 in all cases. 
This means that model 2 is a better fit to the data than the other models. Examination of the fitted parameters for model 2 shows that the parameters associated with $P V\left(t_{b 3}\right.$ and $\left.t_{a 3}\right), C F\left(t_{b 4}\right.$ and $\left.t_{a 4}\right)$, and square items $\left(t_{b 5}\right.$ and $\left.t_{a 5}\right)$ are all small values with small variance, and they are not sensitive to the number of lines serviced by a BRT station. The parameters associated with $t_{0}, t_{b 1}, t_{a 1}$, and exponent terms, $t_{b 2}$ and $t_{a 2}$, are sensitive to the number of lines serviced by a BRT station and they all have large associated variance.
Table 9 Parameters values for model $1(P V \geq 55)$

\begin{tabular}{clllll}
\hline Cases & $t_{0}$ & $t_{b}$ & $t_{a}$ & $t_{v}$ & \multicolumn{1}{l}{$t_{c}$} \\
\hline 1 & 2.227 & 1.284 & 1.105 & 0.286 & -0.039 \\
2 & 8.513 & 0.763 & 0.659 & 0.146 & 0.062 \\
3 & 8.947 & 0.737 & 0.758 & 0.145 & 0.003 \\
$\geq 4$ & 7.407 & 1.584 & 0.843 & 0.175 & -0.131 \\
All data & 6.262 & 1.043 & 0.779 & 0.178 & 0.021 \\
\hline
\end{tabular}

Table 10 Parameters values for model $2(P V \geq 55)$

\begin{tabular}{ccccccccccccc}
\hline Cases & $t_{0}$ & $t_{b 1}$ & $t_{b 2}$ & $t_{b 3}$ & $t_{b 4}$ & $t_{b 5}$ & $t_{a 1}$ & $t_{a 2}$ & $t_{a 3}$ & $t_{a 4}$ & $t_{a 5}$ \\
\hline 1 & 15.122 & -0.098 & -4.302 & 0.008 & -0.004 & 0.562 & 0.822 & -8.556 & 0.002 & 0.051 & -0.052 \\
2 & 3.294 & 2.213 & 25.180 & 0.004 & 0.009 & -0.026 & 1.637 & 2.034 & 0.030 & -0.023 & -0.045 \\
3 & 13.827 & -0.159 & 3.197 & 0.032 & 0.003 & -0.029 & 1.729 & -10.827 & 0.006 & -0.022 & -0.049 \\
$\geq 4$ & 26.505 & -12.649 & -16.061 & 0.166 & -0.075 & -0.096 & 0.816 & 52.537 & -0.001 & 0.057 & -0.033 \\
All data & 10.460 & 0.372 & 4.484 & 0.025 & -0.003 & 0.000 & 1.318 & -8.000 & 0.016 & -0.008 & -0.038 \\
\hline
\end{tabular}

Table 11 Parameters values for model $3(P V \geq 55)$

\begin{tabular}{cccc}
\hline Cases & $t_{0}$ & $b$ & $c$ \\
\hline 1 & 11.910 & 0.068 & 0.009 \\
2 & 11.302 & 0.299 & 0.005 \\
3 & 14.718 & 0.088 & 0.006 \\
$\geq 4$ & 12.645 & 0.115 & 0.008 \\
All data & 12.263 & 0.149 & 0.007 \\
\hline
\end{tabular}

Table 12 Parameters values for model $4(P V \geq 55)$

\begin{tabular}{cccc}
\hline Cases & $t_{0}$ & $t_{b}$ & $t_{a}$ \\
\hline 1 & 8.609 & 1.630 & 1.164 \\
2 & 11.155 & 1.337 & 1.102 \\
3 & 14.911 & 0.911 & 0.951 \\
$\geq 4$ & 13.085 & 1.725 & 0.492 \\
All data & 10.872 & 1.428 & 1.013 \\
\hline
\end{tabular}

In the case when $P V<55$, the $R^{2}$ values are shown in Table 13 and the fitted parameter values are shown as Tables 14-17. The $R^{2}$ values for models 1 and 2 are better than those for models 3 and 4 . However, the fitness $R^{2}$ values of four models are all less than 0.3 with the maximum of 0.267 . When the data is grouped according to the number of BRT lines serviced by a station, the fitness $R^{2}$ values improved significantly.

The comparison of all four models shows that model 2 performs better than the other three models. Recall that model 2 introduced $P V, C F$, exponent of $P B$ and $P A$ and squares of $P B$ and $P A$, and the inclusion of these terms made it possible to better predict dwell time fluctuation.

Table $13 R^{2}$ values for each of four models $(P V<55)$

\begin{tabular}{cccccc}
\hline Models & 1 & 2 & 3 & 4 & All data \\
\hline 1 & 0.365 & 0.235 & 0.364 & 0.694 & 0.266 \\
2 & 0.387 & 0.323 & 0.637 & 0.927 & 0.267 \\
3 & 0.341 & 0.265 & 0.190 & 0.830 & 0.252 \\
4 & 0.362 & 0.235 & 0.351 & 0.475 & 0.261 \\
\hline
\end{tabular}

Table 14 The parameters of model $1(P V<55)$

\begin{tabular}{ccccc}
\hline Cases & $t_{0}$ & $t_{b}$ & $t_{a}$ & $t_{c}$ \\
\hline 1 & 10.145 & 0.561 & 0.662 & 0.034 \\
2 & 10.091 & 1.160 & 0.964 & -0.012 \\
3 & 9.873 & 2.101 & 0513 & 0.238 \\
$\geq 4$ & 18.020 & -1.706 & -0.214 & 0.284 \\
All data & 10.427 & 0.851 & 0.690 & 0.062 \\
\hline
\end{tabular}

Table 15 The parameters of model $3(P V<55)$

\begin{tabular}{cccc}
\hline Cases & $t_{0}$ & $b$ & $c$ \\
\hline 1 & 10.496 & 0.271 & 0.002 \\
2 & 10.716 & 0.493 & 0.006 \\
3 & 11.376 & 0.628 & 0.007 \\
$\geq 4$ & 9.311 & 0.319 & 0.000 \\
All data & 11.165 & 0.348 & 0.005 \\
\hline
\end{tabular}


Table 16 The parameters of model $2(P V<55)$

\begin{tabular}{cccccccccc}
\hline Cases & $t_{0}$ & $t_{b 1}$ & $t_{b 3}$ & $t_{b 4}$ & $t_{b 5}$ & $t_{a 1}$ & $t_{a 3}$ & $t_{a 4}$ & $t_{a 5}$ \\
\hline 1 & 10.774 & 0.343 & -2.252 & 0.008 & 0.053 & -0.016 & -2.055 & 0.000 & -2.297 \\
2 & 13.527 & -0.211 & 22.080 & 0.008 & -0.603 & -0.199 & 5.329 & -0.232 & -0.244 \\
3 & 16.232 & 0.000 & -0.001 & $-8.6 \mathrm{E} 6$ & $6.84 \mathrm{E} 3$ & 0.164 & -26.28 & -0.312 & -0.277 \\
$\geq 4$ & 27.527 & -8.657 & 16.518 & 0.850 & -0.141 & -2.099 & -66.89 & -0.164 & -0.042 \\
All data & 9.705 & 1.048 & 2.573 & 0.006 & -0.015 & 0.777 & 0.846 & 0.000 & -0.002 \\
\hline
\end{tabular}

Table 17 The parameters of model $4(P V<55)$

\begin{tabular}{cccc}
\hline Cases & $t_{0}$ & $t_{b}$ & $t_{a}$ \\
\hline 1 & 10.086 & 0.596 & 0.682 \\
2 & 10.107 & 1.150 & 0.956 \\
3 & 8.083 & 2.792 & 1.108 \\
$\geq 4$ & 9.708 & 0.969 & 0.577 \\
All data & 10.272 & 0.935 & 0.749 \\
\hline
\end{tabular}

\section{Conclusions}

Since it is evident that the conflict between $P A$ and $P B$ impacts dwell time, we introduce this novel term into our non-linear dwell time model. Additionally, exponential terms which account for the number of passengers boarding and alighting, square terms representing $P A$ and $P B$, and the number of standing passengers in vehicles are considered in our model. Our model performs better than both model 3 [9] and model 4 [4].

Overall, the goodness of fit for our model is higher than that of models 3 and 4 . The $R^{2}$ values for our model reach a maximum of 0.831 as shown in Table 9, and a maximum of 0.927 in Table 11 . While a BRT station services one, two or three BRT lines, the maximum values of the fitness $R^{2}$ are $0.680,0.377$ and 0.499 respectively for $P V \geq 55$. For $P V<55$, the maximum values of the fitness $R^{2}$ for those same groups are $0.387,0.323$ and 0.637 , respectively.

In the future, we'll explore the application of this model in automatic vehicle dispatching systems, guided buses, and to help manage multiple BRT vehicles sharing designated roadways. Further research includes the development of the self-adaptive control model for BRT vehicle headway as an extension to the presented dwell model.

\section{Acknowledgements}

We want to express thanks to Yuan XUE and Ke WANG for their help with this work.

This research was supported by the National Science and Technology Support Program of China (No. 2009BAG17B01).

\section{References}

[1] R. Feder, The Effect of Bus Stop Spacing and Location on Travel Time, Pittsburgh: Carnegie Mellon University, 1973.

[2] H.S. Levinson, Analyzing transit travel time performance, Transportation Research Record, 1983, 915: 1-6.

[3] R.P. Guenthner, K. Hamat, Transit dwell time under complex fare structure, Journal of Transportation Engineering, 1988, 114(3): 367-379.

[4] TCQSM, Transit Capacity and Quality of Service Manual, 2nd Edition, 2003.

[5] T. Lin, N.H. Wilson, Dwell time relationships for light rail systems, Transportation Research Record, 1992, 1361: 287-295.

[6] R.P. Guenthner, K.C. Sinha, Modeling bus delays due to passenger boardings and alightings, Transportation Research Record, 1983, 915: 7-13.

[7] A. Puong, Dwell time model and analysis for the MBTA red line, Cambridge: MIT, 2011.

[8] J. Gibson, R. Fernández, A. Albert, Operation formal stops in Santiago, In: Proceedings of the VIII Chilean Congress of Transport Engineering, Santiago, 1997: 397-408.

[9] R. Rajbhandari, S.I. Chien, J.R. Daniel, et al., Estimation of bus dwell times with automatic passenger counter information, Transportation Research Record, 2003, 1841: 120-127.

[10] K.J. Dueker, T.J. Kimpel, J.G. Strathman, et al., Determinants of bus dwell time, Journal of Public Transportation, 2004, 7(1): 21-40.

[11] S. Jaiswal, J.M. Bunker, L. Ferreira, Modeling bus lost time: an additional parameter influencing bus dwell time and station platform capacity at bus rapid transit station platform, In: Transportation Research Board 89th Annual Meeting, Washington D.C., 2010.

[12] J.D. Fricker, Bus dwell time analysis using onboard video, In: Transportation Research Board 90th Annual Meeting, Washington D.C., 2011.

[13] S. Jaiswal, J.M. Bunker, L. Ferreira, Relating bus dwell time and platform crowding at a busway station, In: Proceedings 31st Australasian Transport Research Forum (ATRF), Gold Coast: 239-249.

[14] S. Jaiswal, J.M. Bunker, L. Ferreira. Modeling the Relationships between passenger demand and bus delays at busway stations, In: Transportation Research Board 88th Annual Meeting, Washington D.C., 2009. 